\title{
Calcaneal and Pelvic Fractures: Diagnostic Evaluation by Three-Dimensional Computed Tomography Scans
}

\author{
Michael W. Vannier, Charles F. Hildebolt, Louis A. Gilula, Thomas K. Pilgram, Frederick Mann, \\ Barbara S. Monsees, William A. Murphy, William G. Totty, and Carolyn J. Offutt
}

\begin{abstract}
A major application of three-dimensional (3D) computed tomography (CT) is in the imaging of the skeleton. 3D CT has a potentially important role in determining the presence, type, and extent of fractures, especially of the calcaneus and pelvis. The objective of this study was to compare the diagnostic sensitivity and specificity of 3D CT, CT slices, and plain radiography in the detection and characterization of calcaneal and pelvic fractures. 3D CT reconstructions were obtained by two methods, surface reconstruction and volumetric techniques. Twenty-eight patients were imaged with CT, 3D CT, and plain radiography. The opinion of a musculoskeletal radiologist with access to all images plus clinical history, surgical findings, and follow-up findings was taken as truth. Four additional musculoskeletal radiologists read these cases in a blinded fashion and ranked the modalities with regard to perceived utility. Receiver operating characteristic analysis was used to determine the relative value of each modality in terms of diagnostic quality. All imaging modalities performed comparably in the diagnosis of fractures. CT slices and plain-films were the most useful for more difficult diagnostic tasks such as fracture stability, and the presence of comminution and estimation of the number of fragments. The results suggest that for skeletal areas with complicated anatomy (such as the pelvis and calcaneus), the diagnostic value of $3 D \mathrm{CT}$ is often equivalent to that of conventional methods.
\end{abstract}

Copyright $\odot 1991$ by W.B. Saunders Company

KEY WORDS: three-dimensional computed tomography, computed tomography, fractures, receiver operating characteristic analysis, pelvis, calcaneus

$\mathbf{I}$

N AREAS OF complicated skeletal anatomy such as the pelvis and calcaneus, plain-film radiographs are often of limited diagnostic value because complex three-dimensional (3D) anatomy is compressed into the two dimensions of plain films. Computed tomography (CT) scanning partially overcomes this limitation by representing complex $3 \mathrm{D}$ anatomy with a series of two-dimensional (2D) slices. The number, complexity, and redundancy of these slices can, however, be an obstacle to complete understanding of skeletal anatomy and pathology. Integration of high-resolution axial CT data into 3D images is often preferable to mental integration of 2D images. It improves information transmission to other physicians, and may enhance radiographic diagnosis. ${ }^{1-4}$ Unfortunately, most of the 3D studies that have been reported on the relative performance of plain films, CT scans, and the various $3 \mathrm{D}$ reconstructions for diagnosing fractures of complex anatomical structures have been anecdotal. Our objective was to compare these imaging modalities with regard to their value in the diagnosis of fractures of the pelvis and calcaneus.

\section{MATERIALS AND METHODS}

\section{Patient Population}

We studied calcaneal and pelvic fractures in adults. The details of the sample are shown in Table 1 . Nineteen calcanei were studied in 11 individuals with suspected or overt calcaneal fractures. Thirty-three hemi-pelves with known or suspected fractures were studied in 17 patients. These patients were identified by retrospective analysis of CT scanning logs at the Mallinckrodt Institute of Radiology.

Cases where contiguous slices were obtained through the pelvis and calcaneous, and especially where both sides (injured and uninjured) were included, were given preference. There was bias toward more complex cases because the CT scans had been requested preoperatively, to determine operative approach, procedure selection, or conservative treatment. Fifteen of the pelvic fractures were displaced and 18 were comminuted; 8 of the calcaneal fractures were displaced and 10 comminuted.

\section{Imaging}

Plain films were available for most cases. The images included anterior-posterior (AP) pelvis and frog-leg lateral views (if available) of the hips (Fig 1A), and lateral and tangential (Harris) views of the calcaneus (Fig 4A). The radiographs were selected to be as near to time of injury as possible.

CT scans were available for all cases. For most examinations, $4 \mathrm{~mm}$ slices were taken contiguously through the pelvis or calcaneus at $2 \mathrm{~mm}$ intervals (Figs 1B, 2B). For a

From Mallinckrodt Institute of Radiology, Washington University School of Medicine, St Louis, MO.

Supported in part by Phillips Medical Systems, Inc, under the Pegasus project.

Presented at the 1988 annual meeting of the Radiological Society of North America.

Address reprint requests to Michael $W$. Vannier, $M D$, Mallinckrodt Institute of Radiology, Washington University School of Medicine, 510 S Kingshighway Blvd, St Louis, MO 63110.

Copyright 191991 by W.B. Saunders Company

0897-1889/91/0403-0003\$03.00/0 
Table 1. Sample

\begin{tabular}{|c|}
\hline Calcaneus \\
\hline Patient Population \\
\hline $\begin{array}{l}11 \text { individuals ( } 9 \text { male, } 2 \text { female, } 26-70 \\
\text { years old) }\end{array}$ \\
\hline 8 both feet, 3 single foot $=19$ feet \\
\hline Sample size \\
\hline $\begin{array}{l}N=19 \text { (8 normal, } 11 \text { abnormal) for all but } \\
\text { plain-film }(C T, 3 D, C T)\end{array}$ \\
\hline$N=7$ (1 normal, 6 abnormal) for plain-film \\
\hline Pelvis \\
\hline Patient Population \\
\hline $\begin{array}{l}17 \text { individuals (9 male, } 8 \text { female, } 18-87 \\
\text { years old) }\end{array}$ \\
\hline 16 both hips, 1 single hip $=33$ hips \\
\hline Sample Size \\
\hline $\begin{array}{l}\mathrm{N}=32 \text { (14 normal, } 18 \text { abnormal) for all } \\
\text { but plain-film (CT, 3D, CT) }\end{array}$ \\
\hline $\begin{array}{l}N=27 \text { (11 normal, } 16 \text { abnormal) for } \\
\text { plain-film }\end{array}$ \\
\hline
\end{tabular}

few examinations, $8 \times 8 \mathrm{~mm}$ contiguous nonoverlapping CT sections were obtained. The scans were performed with a Siemens Somatom DR-3 or DR-H CT scanner. CT slice images were recorded on film hardcopy with 15 on 1 format on $14 \times 17$ in sheets of film with a Matrix Instruments Model MI-10 multiformat camera.

\section{D CT Reconstructions}

3D reconstruction of CT scans may be performed by several methods, including depth coding, surface shading, and volumetric techniques. Depth coded images are displayed so that features that are nearest to the observer are lighter and those more distant are darker (Figs 2A, 3A, 5A). This type of $3 \mathrm{D}$ reconstruction was one of the earliest and simplest methods used. ${ }^{5}$ DD depth is still used for mathematical modelling, in calculating areas and volumes, but seldom for display.

Shading techniques provide a better display of surface details, especially those based on computation of the surface gradient. ${ }^{6}$ Using the computed surface gradient map, one may achieve a very detailed surface display (Figs 2B, 3B, 5B). Often, the gradient map is combined with a depth coded image to give a more realistic and complete appearance.

Recently, volumetric techniques that model a data volume as a semitransparent gel have emerged. Volumetric techniques do not explicitly detect surfaces, but simply sum the incremental contribution of each voxel along rays projected in a desired direction to form $3 \mathrm{D}$ views ${ }^{7}$ (Figs $2 \mathrm{C}$, $3 \mathrm{C}, 5 \mathrm{C}$ ). The principal advantages ascribed to $3 \mathrm{D}$ volume images include the ability to visualize subsurface details and retention of small thin structures such as bone and nondisplaced fractures.

Both shaded surface and volume $3 \mathrm{D}$ views ${ }^{2-7}$ were obtained with a PICS-2000 prototype (Philips Medical Systems Inc, Shelton, CT) that incorporates an advanced computer graphics processor unit (Pixar Image Computers, San Rafael, CA), integrated with an applications-oriented medical user's interface running on a Sun 3/180 engineering workstation. The system was configured with a prototype software system intended for 3D osseous and soft tissue imaging called the musculoskeletal package, or MSP.

Depth coded images were produced with software that operates on the scanners that were used in the CT examinations. All 3D CT images were reconstructed as AP, right and left (R\&L) lateral (LAT), R\&L midsagittal, rear, top, and bottom views. These were recorded on gray scale at a 4 on 1 format on $8 \times 10$ in sheets of Kodak GTB film with a Matrix Instruments Model 4007 multiformat camera.

\section{EXPERIMENTAL PROCEDURE}

Truth was established by a musculoskeletal radiologist ( $\mathrm{L}$. Gilula) who reviewed all images, clinical histories, and follow-up examinations. In many of the cases the follow-up exams included plain-films that could be used to confirm diagnoses, through callus formation at questionable nondisplaced sites in particular. The individual who established truth did not participate in the rating and ranking tests that were used to assess diagnostic performance.
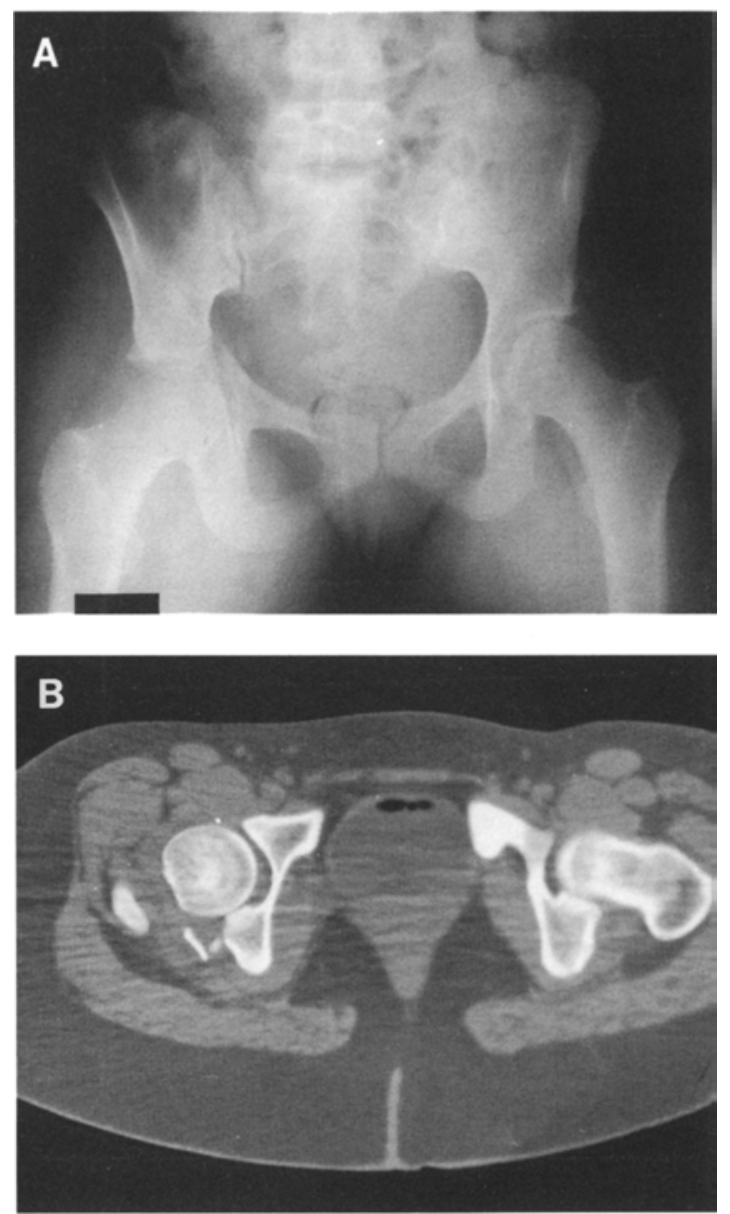

Fig 1. (A) AP radiograph of the pelvis shows acetabular fracture of the patient's right hip. (B) A CT slice shows multiple bony fragments adjacent to the right acetabulum. 


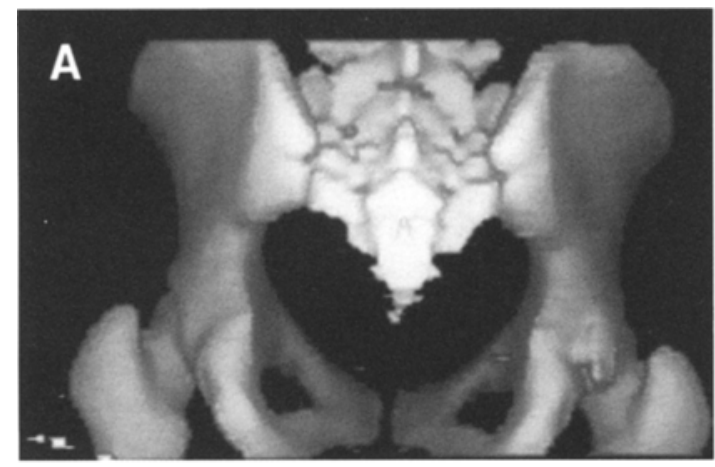

Fig 2. (A) This is a posterior-anterior view obtained with 3D CT and depth coding that shows the posterior rim acetabular fracture of the right hip. (B) A gradient shaded surface view shows an irregular groove at the acetabulum. (C) A postero anterior volumetric view in the same patient shows a lucency in the right acetabulum at the fracture site.
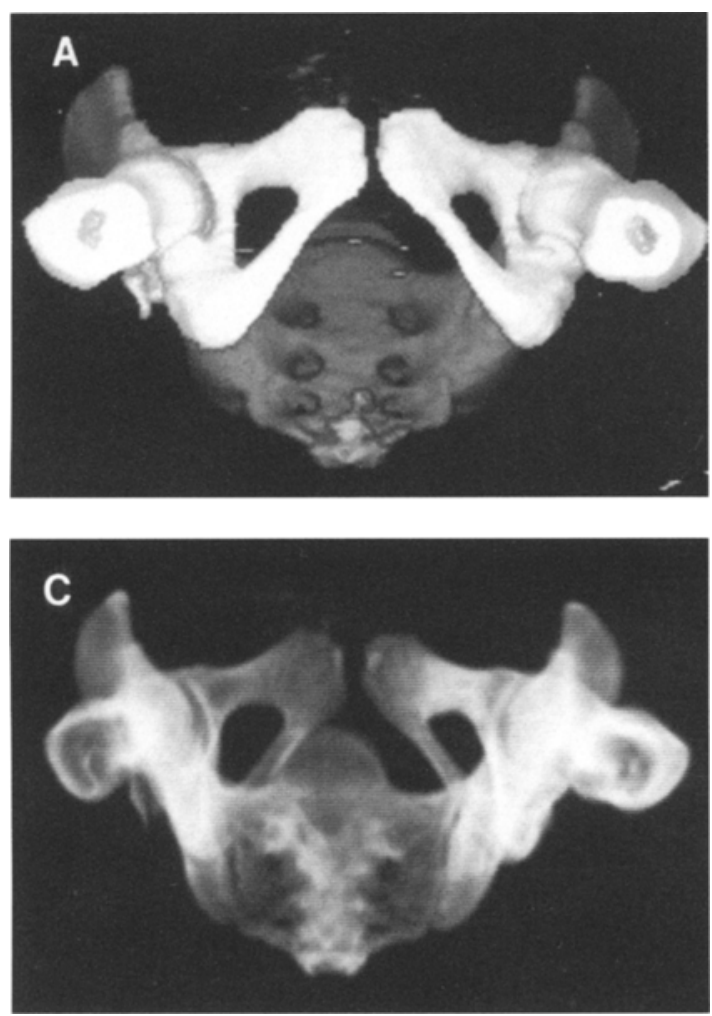
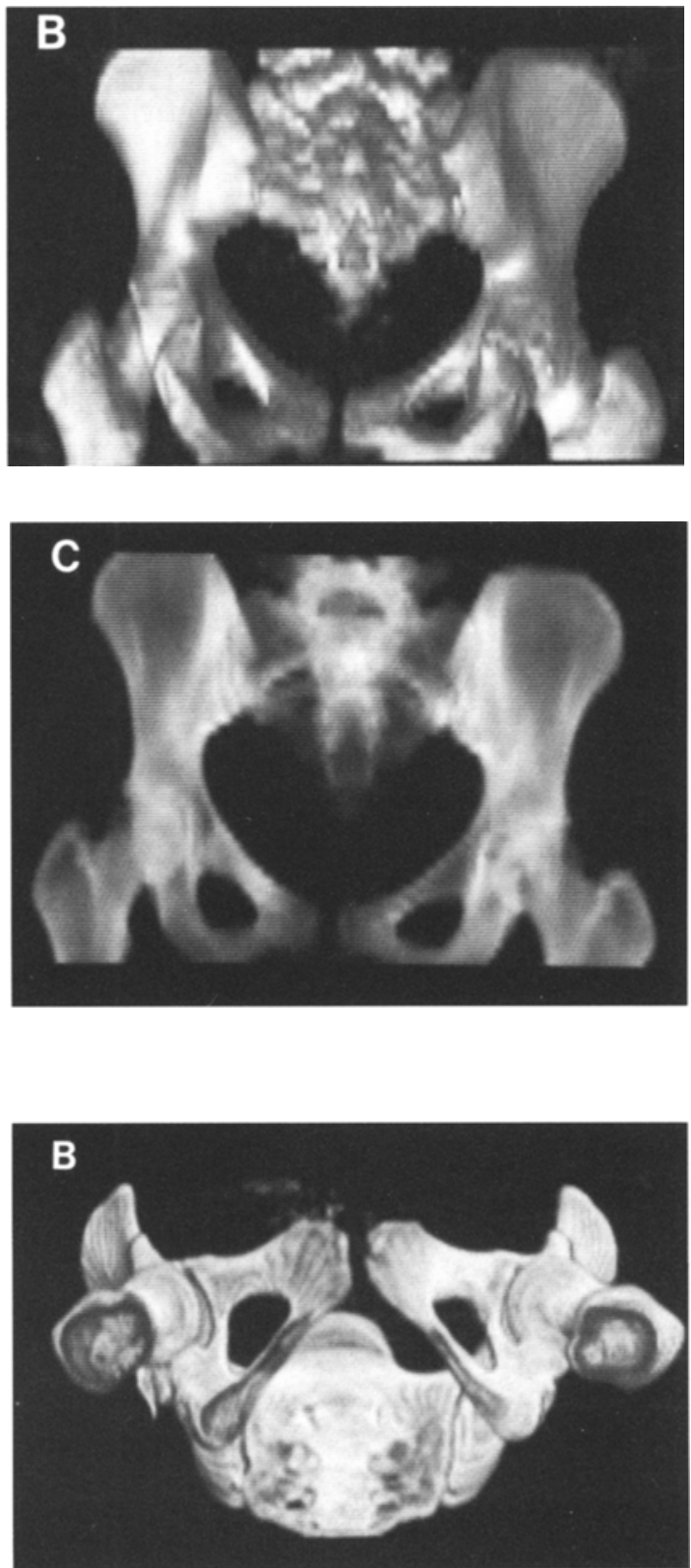

Fig 3. (A) A worm's eye view of the pelvis, from the caudalcranial projection shows a right acetabular fracture with bony fragments posterior to the joint. (B) The gradient surface shaded view from the caudal-cranial projection shows the bony fragments. (C) A volumetric view from the same perspective illustrates the transparency that can be achieved. 

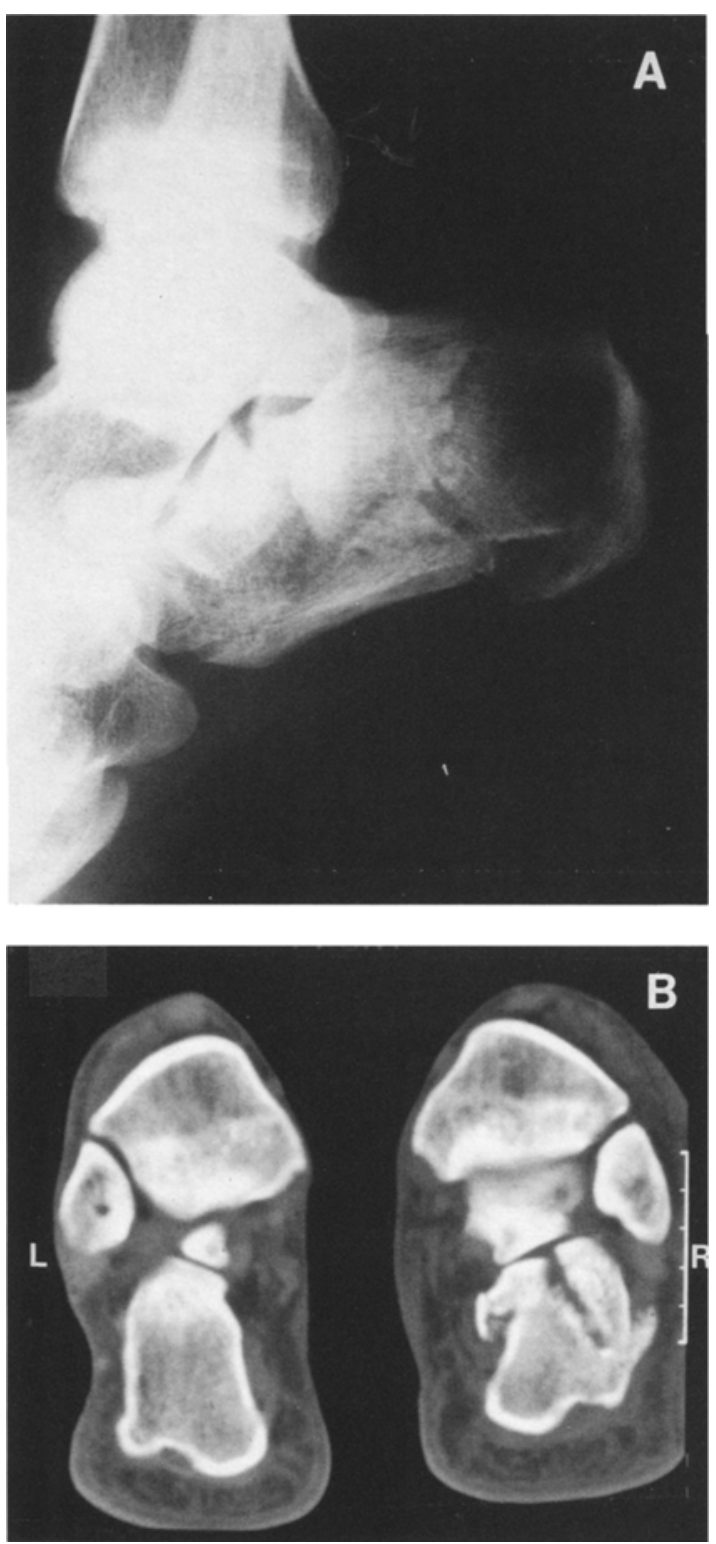

Fig 4. (A) A lateral radiograph of the ankle shows a comminuted fracture of the calcaneus. (B) Transaxial CT slices of both ankles show the comminuted right calcaneal fracture with associated impaction and soft tissue swelling.

The observers who ranked and rated images were all experienced radiologists from the Musculoskeletal Radiology Section of the Mallinckrodt Institute of Radiology. For presentation to the observers, all patient information and the date of imaging were masked with black photographer's tape. The cases were identified only with a number assigned sequentially in the course of copying and archiving for the experiments.

First, shaded and volumetric $3 \mathrm{D}$ reconstructions were rank ordered by the observers. Selected cases were presented and each observer asked separately which set of images (shaded or volumetric) appeared to be of the most value for diagnosis of fractures. Depth coded 3D surface reconstruction were found to be inferior to other $3 \mathrm{D}$ techniques for diagnosis of sutural synostosis in an earlier study, ${ }^{8,9}$ so we did not include $3 \mathrm{D}$ depth images in the ranking experiment.

The rating study was performed using all imaging modalities. Films for each modality were loaded onto a rotating viewer with fluorescent illumination and rated response forms provided to each observer for each case reviewed. Rated responses were on a 5-point scale that varied from " 1 " (definitely or almost definitely a fracture) to "5" (definitely or almost definitely no fracture).

\section{ANALYSES}

ROC curves ${ }^{10-16}$ and areas beneath the curves $\left(A_{2}^{17-19}\right)$ were calculated with the ROCFIT program, ${ }^{20,21}$ using data pooled from all observers; that is, each rating by each observer was counted as a separate case. ${ }^{22}$ Data were pooled because of the small sample sizes and because the data were degenerate in some instances. ${ }^{21.22}$ The degeneracy was caused by consistently high levels of certainty in the observers' responses, both correct and incorrect, which resulted in the fitted curves becoming vertical or horizontal at some false positive fraction. By pooling the responses from all observers, the problem with degenerate data was partially overcome. In addition, to more fully compare the diagnostic values of the imaging modalities in cases with degenerate data, sensitivity $(\mathrm{TP} /(\mathrm{TP}+\mathrm{FN}))$, specificity $(\mathrm{TN} /(\mathrm{FP}+$ $\mathrm{TN}))$, and accuracy $(\mathrm{TP}+\mathrm{TN}) /(\mathrm{TP}+\mathrm{TN}+\mathrm{FP}+\mathrm{FN})$ (where $\mathrm{T}=$ true, $\mathrm{F}=$ false, $\mathrm{P}=$ positive, and $\mathrm{N}=$ negative) were calculated.

Frequency distributions were used to display observer responses for the number of fragments in comminuted fractures. Patients were divided into groups on the basis of the true number of fragments and the frequency of observer responses for each fragment-number category $(1,2,3,4$, $5-10,10-15,15+)$ calculated for each group. Again, data were pooled for all observers, in this case because the sample size had been substantially reduced by restricting data to subgroups of comminuted fractures based on the number of fragments.

\section{RESULTS}

Rankings of perceived diagnostic value were quite different for pelvic and calcaneal fractures. The observers ranked $3 D$ volume and $3 D$ shaded images nearly equal in subjective diagnostic utility for images of the calcaneus (Fig $6 \mathrm{~A}$ ), but overwhelmingly preferred 3D volume images for diagnosing pelvic fractures (Fig 6B).

These patterns were not repeated in the ROC analyses of diagnostic utility. For diagnosing presence or absence of a fracture of the calcaneus, 3D shaded was superior to $3 \mathrm{D}$ volume (Table 2, Figure 7A), in contrast to their nearly equal subjective rankings. For fractures of the pelvis, 3D shaded and 3D volume images 

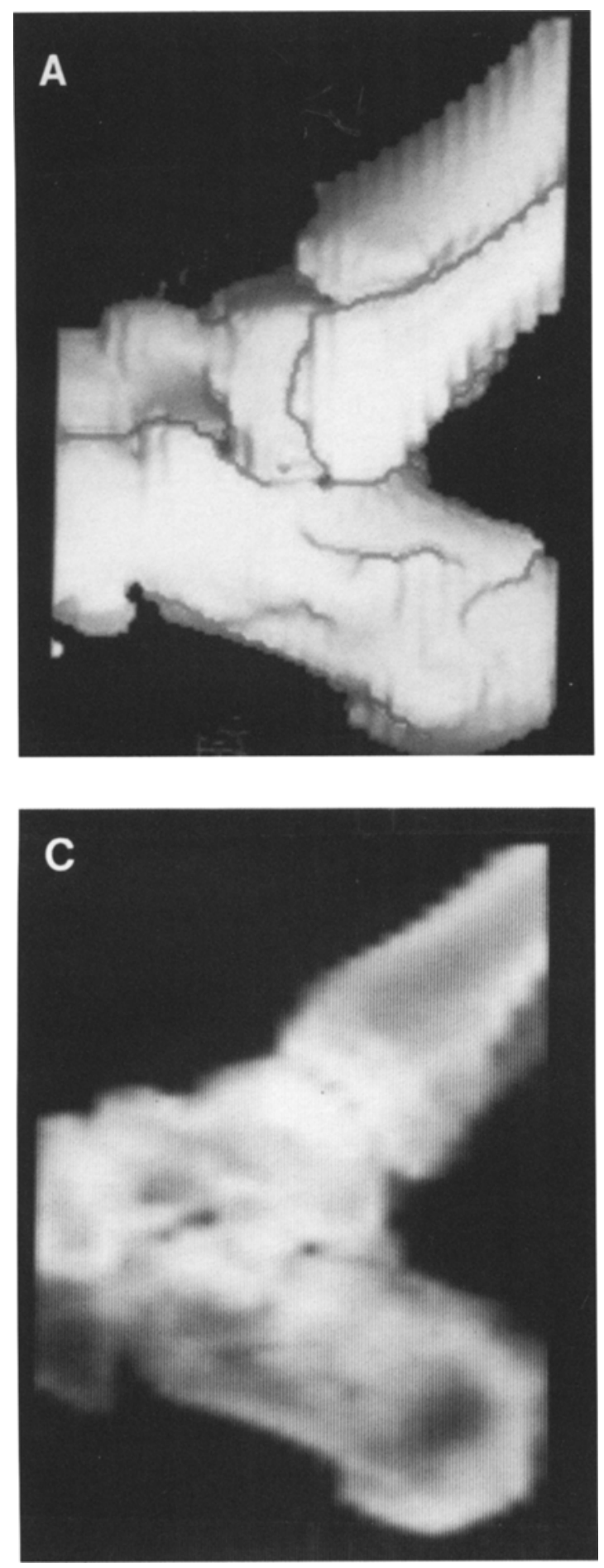

were objectively equal (Table 3 , Fig 7B) for diagnosis, in contrast to the subjective preference for 3D volume.

All imaging modalities were nearly equivalent for diagnosing the presence or absence of a

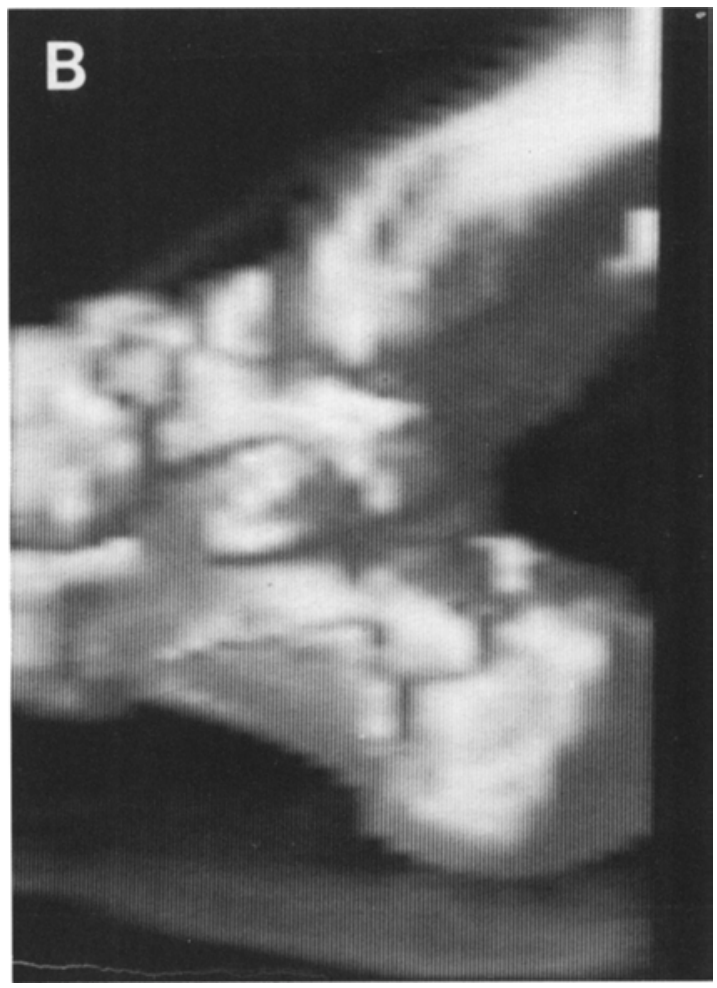

Fig 5. (A) A lateral view of the ankle with depth coding shows a prominent slice artifact from transaxial CT scanning. The fracture fragments appear almost fused in this interpolated depth coded image. (B) A gradient shaded view illustrates the fracture lines at the surface of the calcaneus with associated deformity. (C) The volumetric image provides a suggestion of multiple fracture fragments but little definition of their outlines.

fracture of both the pelvis and the calcaneus (Fig 7, Tables 2, 3). All areas under the ROC curves were within $10 \%$ of the best one.

The clearest differences between modalities is in the shape of the ROC curves. In particular, 

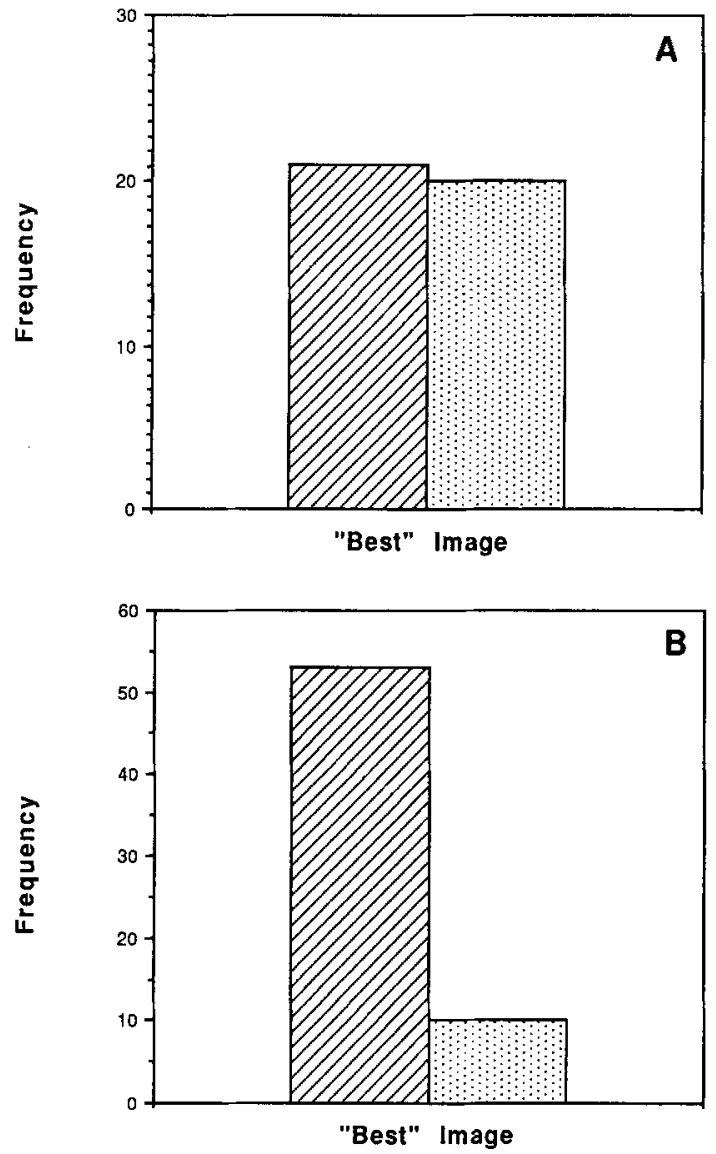

Fig 6. Results of subjective rankings of 3D volumetric and shaded image quality for (A) calcaneus and (B) pelvis. 30 volume; 圈 3D shaded.

the sharpness of the curve in the transition from vertical to horizontal is strongly controlled by the "confidence" of the observer, as expressed in the number of "definite" ("definitely or almost definitely a fracture" or "definitely or almost definitely no fracture") responses. CT slice and plain-film have distinct "corners" joining the nearly vertical and horizontal lines produced by consistently definite responses.

To make a meaningful distinction between the diagnostic utility of the various imaging modalities, a number of more difficult diagnostic tasks were examined. These included (1)

Table 2. Calcaneal Fracture (All Observers)

\begin{tabular}{lcccc}
\hline Imaging modality & Area & \multicolumn{1}{c}{ Accuracy $(\%)$} & Sensitivity $(\%)$ & Specificity $(\%)$ \\
\hline Plain-film & - & 96.2 & 95.8 & 100.0 \\
3D shaded & 0.958 & 92.1 & 90.7 & 93.9 \\
3D depth & 0.943 & 86.5 & 87.8 & 84.8 \\
CT slice & 0.911 & 90.7 & 85.7 & 97.0 \\
3D volume & 0.867 & 84.0 & 79.6 & 90.3 \\
\hline
\end{tabular}

fracture stability, (2) presence of an intraarticular component or fragments in the joint space, (3) comminution, and (4) number of fragments in a comminuted fracture. Because the numbers of responses for these tests were limited to correctly identified fractures, analyses were restricted to pelvic images, for which the number of patients was greatest.

Plain-film images were markedly superior for diagnosis of fracture stability (Table 4, Fig 8A), followed by $\mathrm{CT}$ slice, 3D volume and 3D shaded (which were equal in utility), and $3 \mathrm{D}$ depth that markedly was inferior to the rest. CT slice and plain-film were equal, and best, for diagnosing an intraarticular component to a fracture (Table 4, Fig 8B). These modalities were closely followed by $3 \mathrm{D}$ volume and $3 \mathrm{D}$ shaded, also
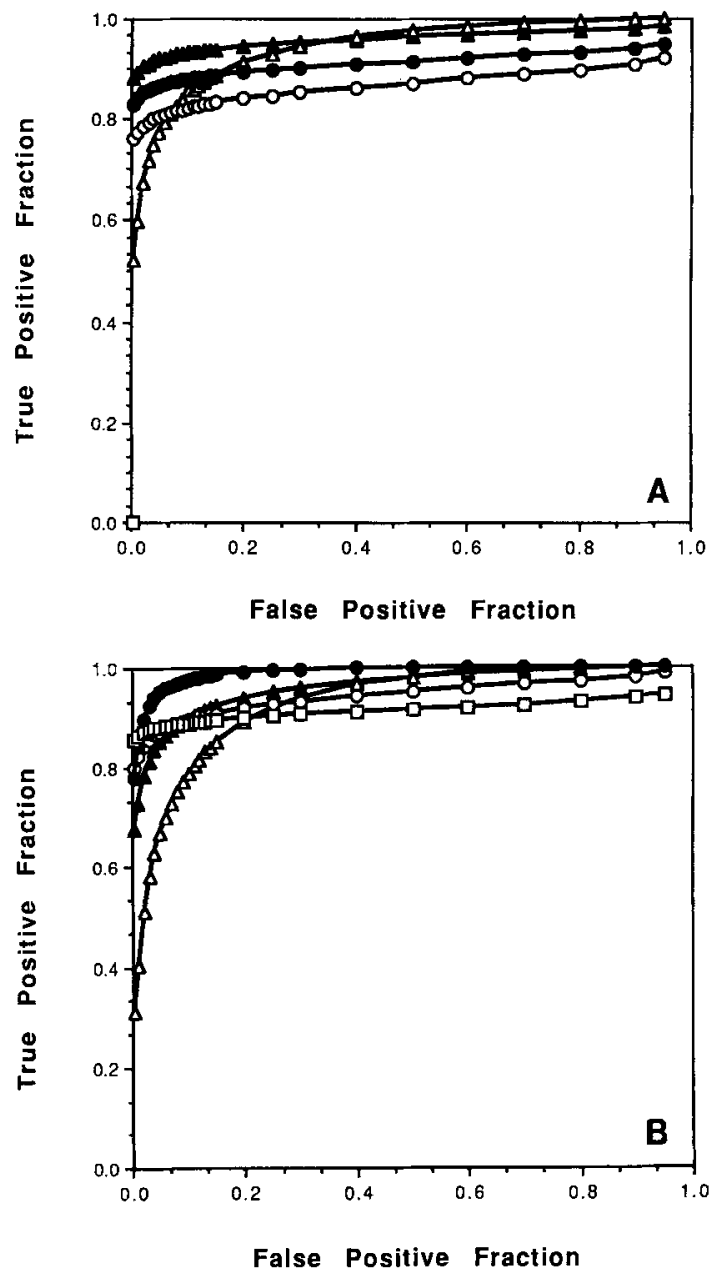

Fig 7. ROC curves for detection of fractures involving the (A) calcaneus and (B) pelvis. (All observers) $\triangle$ 3D shaded; $\triangle$ 3D depth; $O$ 3D volume; $\rightarrow$ CT slice; $\square$ plain film. 
Table 3. Pelvic Fracture (All Observers)

\begin{tabular}{ll}
\hline Imaging Modality & Area \\
\hline CT slice & 0.990 \\
3D shaded & 0.961 \\
3D volume & 0.944 \\
3D depth & 0.930 \\
Plain-film & 0.915 \\
\hline
\end{tabular}

equal, and 3D depth that was again markedly inferior to other modalities.

No imaging modality was consistently in agreement with "truth" for the identification of bone fragments in the joint space (Table 4, Fig 8C). If one considers false-positive fractions of less than 0.2 , none of the modalities perform much better than would be expected by chance. CT slice and plain-film were again nearly tied for best, though they were followed very closely by $3 \mathrm{D}$ shaded. 3D depth and 3D volume were tied and markedly inferior to the rest.

CT slice was best for identifying comminution, followed by plain-film (Table 4, Fig 8D). 3D shaded and 3D depth were tied and inferior, with $3 \mathrm{D}$ volume having the least diagnostic value. Overall, none of the modalities performed well in the diagnosis of comminution. Even CT slice and plain-film, which performed

Table 4. Pelvic Fracture (All Observers)

\begin{tabular}{|c|c|c|c|c|}
\hline Imaging Modality & Area & Accuracy & Sensitivity & Specificity \\
\hline Stable & & $(\%)$ & $(\%)$ & $(\%)$ \\
\hline Plain film & 0.833 & - & - & - \\
\hline CT slice & 0.696 & - & - & - \\
\hline 3D volume & 0.688 & - & - & - \\
\hline 3D shaded & 0.688 & - & 一 & - \\
\hline 3D depth & 0.499 & - & - & - \\
\hline \multicolumn{5}{|l|}{ Intra-articular } \\
\hline CT slice & 0.869 & - & - & - \\
\hline Plain film & 0.867 & - & 一 & - \\
\hline $3 \mathrm{D}$ volume & 0.815 & - & - & - \\
\hline 30 shaded & 0.796 & - & - & - \\
\hline 3D depth & 0.611 & - & - & - \\
\hline \multicolumn{5}{|c|}{ Fragments in Joint Space } \\
\hline CT slice & 0.762 & - & - & - \\
\hline Plain film & 0.754 & - & - & - \\
\hline 3D shaded & 0.721 & - & - & - \\
\hline 3D depth & 0.636 & - & - & - \\
\hline 3D volume & 0.632 & - & - & - \\
\hline \multicolumn{5}{|l|}{ Comminuted } \\
\hline CT slice & - & 84.3 & 86.6 & 33.3 \\
\hline Plain film & - & 76.3 & 76.8 & 66.7 \\
\hline 3D shaded & 0.630 & 48.0 & 46.3 & 62.5 \\
\hline 3D depth & 0.600 & 50.7 & 50.8 & 50.0 \\
\hline 3D volume & 0.459 & 61.2 & 63.1 & 0.0 \\
\hline
\end{tabular}

best, had specificities of only $33 \%$ and $67 \%$, respectively.

When observers correctly identified a comminuted fracture, imaging modalities differed in their usefulness for determining the number of fragments (Fig 9). Plain-film appeared to be best when there were fewer than five fragments, and CT slice best with more than five. Observers consistently underestimated the number of fragments when using 3D shaded images and were inconsistent when using 3D depth and volume.

In general, statistical tests for significances were of limited value because of small sample sizes and low power, with the accompanying high likelihood for committing Type I and Type II errors. Moreover, the results revealed no clear patterns so tests were not needed for hypotheses of differences.

\section{DISCUSSION}

No objective, comparative diagnostic evaluation of diagnostic imaging methods for pelvic and calcaneal fractures has been reported. This study was performed to assess the relative value of plain-films, CT slices and 3D CT reconstruction images for fracture diagnosis.

These results show all imaging modalities to be highly capable at identifying presence/ absence of a fracture, and CT slice and plainfilm most useful for more difficult diagnostic tasks such as fracture stability, the presence of comminution, and estimation of the number of fragments. Of the 3D reconstructions, shaded and volume rendering appear markedly superior to depth, with shaded having a slight advantage over volume when all tests are considered.

The superiority of plain-film and CT slice may be due, to some degree, to familiarity, as $3 \mathrm{D}$ reconstructions were new to some of the observers. This familiarity may also have resulted in high levels of observer confidence that in turn caused the sharp "corners" on many of the CT slice and plain-film ROC curves.

Another possible explanation for the seeming superiority of plain-film and CT slice for diagnosis is that the radiologist responsible for determining "truth" reported that his subjective impression was that he gave most weight to these modalities in his decision. The good 

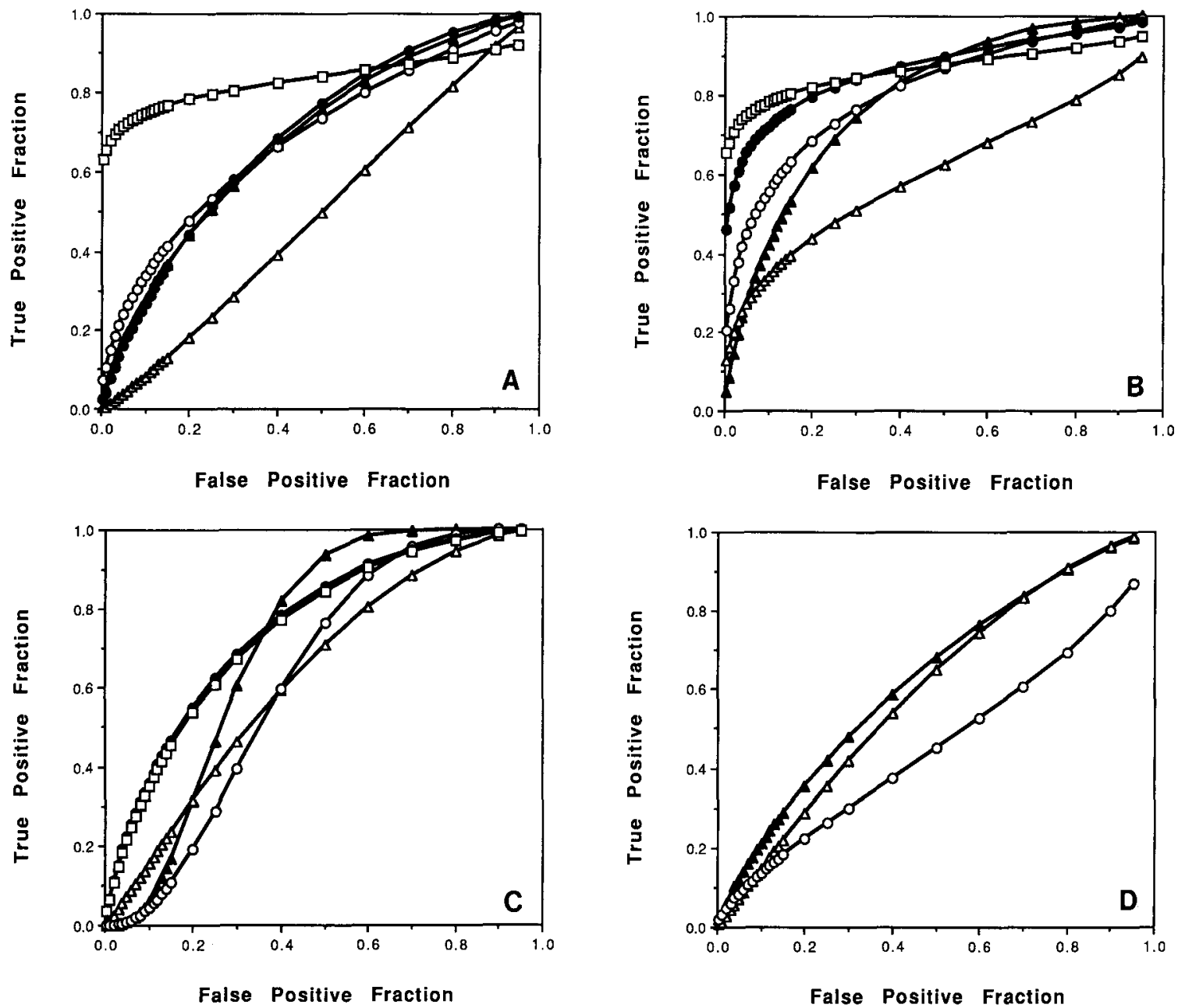

Fig 8. ROC curves for pelvic fracture characteristics obtained from pooled data from four observers. (A) Fracture stability (all observers). (B) Presence of intra-articular extension of the fracture (all observers). (C) Presence of fracture fragments within the joint space (all observers). (D) Comminution (all observers). $\triangle$ 3D shaded; $\triangle$ 3D depth; $O$ 3D volume; $\bigcirc \mathrm{CT}$ slice; $\square$ plain film.

performance of plain-film and CT slice may therefore be simply a reflection of agreement between the observers and the investigator who established truth, and not an indication of diagnostic preeminence.

Among the most interesting results of this study is the great disparity between the subjective rankings of images and the objective ROC evaluations of diagnostic utility. For pelves, observers overwhelmingly preferred $3 \mathrm{D}$ volume to 3D shaded images, but their actual diagnostic performance was slightly better with 3D shaded. For calcaneal fractures, observers slightly preferred $3 \mathrm{D}$ volume images, whereas their performance was markedly better with 3D shaded. Perhaps the "transparent" impression of 3D volume mimics the more familiar plain-films and imparts some of the confidence associated with their use.

In addition, it is worth noting that diagnosis is not the only use for body imaging. It can play an important role in treatment planning and assessment as well, especially when surgery is required. It may well be that $3 \mathrm{D}$ reconstructions, of which shaded and volume are the best for diagnosis, are more useful than conventional imaging modalities when one considers the ease with which information can be transmitted from the radiologist to the surgeon. In addition, the surgeon may be better able to plan surgery based on images that closely resemble what will be seen in the operating room, rather than on images (CT slices) that he or she must mentally reformat in his or her mind, or those (plain 


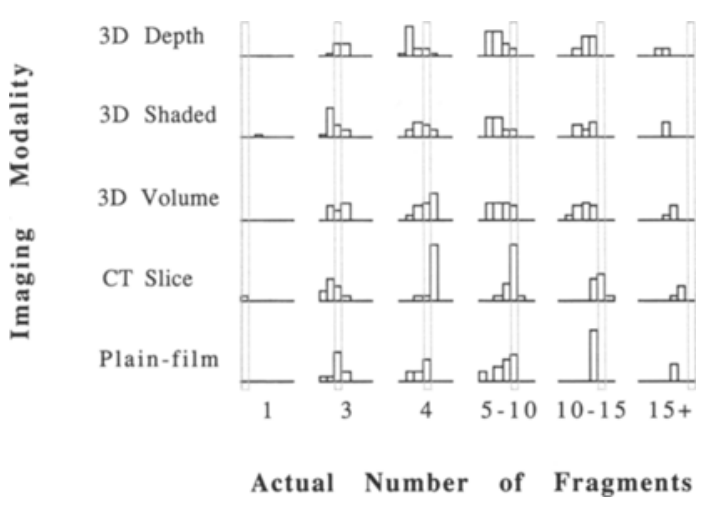

Fig 9. The number of fracture fragments in comminuted pelvic fractures was estimated by four observers. These results are presented as histograms of the number of fragments estimated with truth highlighted. There were no images with the actual number of fragments equal to two.

films) from which he or she must extrapolate 3D anatomy.

\section{LIMITATIONS}

Truth was based primarily on analysis of images. This may have played a role in the good performance of plain-film and CT slices. This methodologic bias could be overcome by creating fractures under controlled conditions in fresh cadavers, radiographing the fracture sites, and basing truth on prosecting and defleshing of the cadavers.

Moreover, this study was retrospective in nature, and the patients were not examined under strict protocol conditions with identical imaging techniques used in every case. The order in which examinations were administered was nonrandom. The sample size was small with an over-representation of abnormals. The quality of examinations, especially for the 3D reconstructions, was nonuniform due to the improvements in this technology that have taken place over the past 5 years.

We presented only monochrome, static selected views, and especially for $3 \mathrm{D}$ reconstructions, there may be significant advantages for color display and dynamic rotations. This remains to be evaluated in future studies.
Finally, all observers were very experienced with plain-film radiography and conventional slice-type CT scanning, and incremental improvement available with other modalities may be masked, to some extent, by observer learning.

\section{CONCLUSIONS}

All modalities performed comparably for the identification of fractures. For more difficult diagnostic tasks such as the determinations of (1) stability, (2) fragment presence or absence in the joint space, (3) comminution, and (4) the number of fragments, plain-film and CT slice were generally superior to other techniques. 3D shaded was the most useful of the $3 \mathrm{D}$ reconstruction techniques, but only slightly superior to 3D volume.

The objective results for the $3 \mathrm{D}$ modalities were opposite to the subjective rankings of volumetric and shaded 3D images based upon perceived diagnostic value. For subjective rankings, observers expressed a marked preference for volumetric images.

Because of the small sample size and problematic considerations in the establishment of truth, the above conclusions are tentative. Considering the seemingly comparable value of $3 \mathrm{D}$ reconstructions to traditional imaging modalities for the diagnosis of fractures of complex anatomical structures, the ability of $3 \mathrm{D}$ reconstructions to convey information on complex anatomy from the radiologist to the clinician, and the similarity of $3 \mathrm{D}$ reconstructions to what is seen by the surgeon in the operating room, a full study of the utility of 3D CT in the diagnosis of fractures is warranted.

\section{ACKNOWLEDGMENT}

Technical assistance from Thomas Giordano, Robert Drebin, Claire Eurglines, and Mickey Mantle are gratefully appreciated. The assistance of Siemens Medical Systems Inc, Iselin, $\mathrm{NJ}$, is appreciated. The ROC programs we used were provided by Professor C.E. Metz and J. Shen of the University of Chicago. Manuscript preparation by M. Akin is gratefully appreciated.

\section{REFERENCES}

1. Totty WG, Vannier MW: Complex musculoskeletal anatomy: Analysis using three dimensional surface reconstruction. Radiology 150:173-177, 1984
2. Fishman EK, Drebin RA, Magid D: Volumetric rendering techniques: Applications for 3-dimensional imaging of the hip. Radiology 163:737-738, 1987 
3. Fishman EK, Magid D, Ney DR, et al: Three dimensional imaging in orthopedics: State of the art 1988. Orthopedics 11:1021-1026, 1988

4. Fishman EK, Magid D, Ney DR, et al: Threedimensional imaging and display of musculoskeletal anatomy. Jour Comp Asst Tomogr 12:465-467, 1988

5. Vannier MW, Marsh JL, Warren JO: Three dimensional CT reconstruction images for craniofacial surgical planning and evaluation: Radiology 150:179-184, 1984

6. Drebin RA, Carpenter LA, Hanrahan P: Volumetric rendering. Computer Graphics 22:65-74, 1988

7. Trivedi SS: Volumetric imaging for medical applications. Cemax Inc, 1987

8. Vannier MW, Hildebolt CF, Marsh JL, et al: Craniosynostosis: Diagnostic value of three-dimensional CT reconstruction. Radiology 173:669-673, 1989

9. Pilgram TK, Vannier MW, Hildebolt CF, et al: Craniosynostosis: Image quality, confidence, and correctness in diagnosis. Radiology 173:675-679, 1989

10. Metz CE: ROC methodology in radiologic imaging. Invest Radiol 21:720-733, 1986

11. Metz CE: Basic principles of ROC analysis. Semin Nuc Med 8:283-298, 1987

12. Swets JA: ROC analysis applied to the evaluation of medical imaging techniques. Invest Radiology 14:109-121, 1979

13. McNeil BJ, Hanley JA: Statistical approaches to clinical predictions. N Engl J Med. 304:1292-1294, 1981
14. McNeil BJ, Hanley JA: Statistcal approaches to the analysis of receiver operating characteristic (ROC) curves. Med Dec Making 4:137-150, 1984

15. Swets JA: Form of empirical ROCs in discrimination and diagnostic tasks: Implications for theory and measurement of performance. Psychol Bulletin 99(2):181-198, 1986

16. Getty DJ, Pickett RM, D'Orsi CJ, et al: Enhanced interpretation of diagnostic images. Invest Radiol 23:240252,1988

17. Hanley JA, McNeil BJ: A method of comparing the areas under receiver operating characteristic curves derived from the same cases. Radiology 148:839-843, 1983

18. Hanley JA, McNeil BJ: Maximum attainable discrimination and the utilization of radiologic examinations. $J$ Chron Dis 35:601-611, 1982

19. Hanley JA, McNeil BJ: The meaning and use of the area under a receiver operating characteristic (ROC) curve. Radiology 143:29-36, 1982

20. Metz CE, Wang DL, Kronman HB: A new approach for listing the significance of differences between ROC curves measured from correlated data, in: Deconink F (ed): Information Processing in Medical Imaging. The Hague, Nijhoff, 432-445, 1984

21. Metz CE: Some practical issues of experimental design and data analysis in radilogical ROC studies. Invest Radiol 24:234-245, 1989

22. Swets JA, Pickett RM: Evaluation of diagnostic systems. New York, NY, Academic Press, 1982 